\title{
Ultrasonography in diagnostic dermatology: a primer for clinicians
}

\author{
Sami Raza ${ }^{1} \cdot$ Faisal Ali $^{2,3} \cdot$ Firas Al-Niaimi $^{4}$ \\ Received: 8 December 2020 / Revised: 2 June 2021 / Accepted: 16 November 2021 / Published online: 9 January 2022 \\ (c) The Author(s), under exclusive licence to Springer-Verlag GmbH Germany, part of Springer Nature 2022
}

\begin{abstract}
Ultrasound has long been a diagnostic staple throughout medicine, with extensive use in orthopaedics and obstetrics. In this review, we find there are a range of versatile uses for diagnostic ultrasound in dermatology, with particular clinical relevance in the imaging of skin tumours. Our review outlines that diagnostic ultrasound can play an important role as an adjunct, but shortfalls remain with inter- and intra-operator discrepancies in aptitude and accuracy.
\end{abstract}

Keywords Basal cell carcinoma $\cdot$ Dermatology $\cdot$ Diagnosis $\cdot$ Imaging $\cdot$ Squamous cell carcinoma $\cdot$ Ultrasound

\section{Introduction}

Imaging has a long heritage of use in dermatology, particularly for the characterisation of benign lesions. Recent innovations in ultrasound technology have refocused attention on both diagnostic and therapeutic applications. In this review, we outline the physics underlying the use of ultrasonography in diagnostic dermatology, radiological features of the skin lesions commonly investigated by ultrasonography, including physiological structures, skin cancers, vascular lesions, benign skin lesions, and inflammatory dermatoses.

\section{Basics of ultrasound physics}

The ultrasound image is generated when mechanical oscillations of a crystal are stimulated by electrical pulses, which is known as the piezoelectric effect [1]. When the transducer is placed onto the skin, the ultrasound is reflected by deeper structures in the skin and deeper plane. The acoustic waves are then translated into electrical energy, which appear on the monitor. The resolution of the image is

Firas Al-Niaimi

firas55@hotmail.com

1 University of Birmingham, Birmingham, United Kingdom

2 Mid Cheshire Hospitals NHS Foundation Trust, Macclesfield, United Kingdom

3 St John's Institute of Dermatology, London, United Kingdom

4 Aalborg University, Aalborg, Denmark affected by wavelength; higher frequencies produce shorter wavelengths, which in turn lead to better resolution, at the expense of reduced penetration [2]. Consequently, to image deeper penetrating structures in the skin, this will need to be at a lower resolution.

Ultrasound waves can be refracted, deflected, absorbed, or reflected. They are dependent on three key factors which affect the intensity of the acoustic impulse: acoustic density ( $Z$; which is the resistance to sound wave propagation), sound velocity of adjacent tissues $(C)$ and the product of density $(\rho)$ [3]. The acoustic density of structures determines the proportion of sound beams that are returned to the transducer; a moderate difference in acoustic density will result in the beam being reflected and returned to the transducer, with the remaining beams propagated or transmitted into deeper tissue layers. In the context of a higher acoustic density, there is a higher intensity of the wave reflected back to the transducer and a proportional decrease of the wave transmitted or propagated [1].

Echogenicity is related to the speed upon which the ultrasound waves can be propagated and returned to the transducer and is dictated by structures in each layer of the skin: keratin in the epidermis, collagen in the dermis and fat in the subcutaneous layer. Hyperechoicity correlates with high intensity echoes and vice versa for hypoechoicity. The epidermis can be visualised as a hyperechoic line and the dermis as a hyperechoic band [2]. The papillary layer of the dermis has reduced echogenicity due to its thinner nature and a reduced amount of collagen fibres [4]. Owing to ageing skin or excessive UV exposure, a sub-epidermal low-echogenic band [4] may be visualised and can act as a 
surrogate marker for the consequences of photo-aging [5]. Appendages appear as well-demarcated anechoic structures, whilst vessels, connective tissue and fascia in the subcutaneous layer are hyperechoic [3].

To ensure standardisation of ultrasound procedures, a working group [6] suggested using a minimum frequency of $15 \mathrm{MHz}$ and to use colour Doppler ultrasound, which helps differentiate between types of lesions.

There is, however, a wide pricing discrepancy between different types of ultrasound units. Whilst small, point-ofcare units can be cheap, more premium ultrasound machines, particularly those that are larger in size and can produce higher quality images, can cost in excess of $€ 100,000$. Given the recent backdrop of the COVID-19 pandemic and its potential to reduce healthcare budgets, healthcare commissioners and dermatologists should seek to select the most appropriate device in terms of image clarity and price, as there is an inevitable trade-off between pricing and clinical efficacy.

\section{Applications in dermatology}

There are a wide variety of dermatological applications of ultrasound.

\section{Lymph nodes}

Ulrich et al. [3] noted that a frequency between 7.5 and $15 \mathrm{MHz}$ is ideal to detect regional lymph nodes. Normal lymph nodes that are not enlarged are not detectable on ultrasound due to a similar echo identity to the surrounding adipose tissue. Regional lymph nodes demonstrate an oval bi-zonal architecture, a cellular outer region with lymph follicles which produces a hypoechoic structure; the inner zone is hyperechoic due to connective tissue and vasculature. Axillary and inguinal lymph nodes demonstrate a three-layered morphology with the adipose tissue, producing a hypoechoic core. A metastatic lymph node is round, with a hypoechoic centre and necrosis, whereas an inflamed variant is elliptical with a hyperechoic centre and central vascularisation.

\section{Scars}

A clinical trial [7] sought to investigate a new method to reduce acne scarring. 19 patients with different degrees of acne scarring were recruited to assess unipolar fractional ablative radiofrequency with acoustic pressure ultrasound guided injection of a dermal injection of a preparation for the treatment of scars. The bioactive compounds in the injection included molecules that had anti-inflammatory, whitening, and keratolytic properties. Patients had four treatment sessions at 3-week intervals. The results indicated significant improvement in acne scarring on the face, back, and shoulders $(p<0.0001)$, with a progressive fading in total scarring on the face and back and shoulders at 2 months (57\%; 49\%) and 6 months $(62 \% ; 58 \%)$, with patients generally satisfied with their results. The authors concluded that this is an effective method to reduce acne scarring. This trial underlines the importance of dermatologists being adept at using ultrasound to treat a common sequela of acne to deliver both clinical and psychological results.

Lucas et al. [8] have identified the relevance of ultrasound in patients with cosmetic scars. Hypertrophic scars are possible post-operative complications attributed to increased collagen deposits. In one study, ultrasound was a reliable tool in differentiating the scar from healthy tissue with the added benefit that ultrasound, when compared to photographs in assessing wound healing following punch biopsy wounds, allowed to assess for any deep structural wound changes, whereas photography would only permit superficial assessment. In terms of wound healing, a separate study found ultrasound was effective in showing differences between healing wounds and those that were non-healing. This could potentially allow investigating deep tissue structures without biopsies.

\section{Dermal oedema}

In a comparison of ultrasound against magnetic resonance imaging in the assessment of dermal water [8], the authors concluded that ultrasound is more sensitive in assessing dermal oedema and is useful to assess conditions presenting with oedema, such as urticaria.

\section{Skin tumours}

In principle, ultrasound utilisation in skin cancer straddles diagnosis, staging and treatment phases, and presents an opportunity to integrate this into the current workup of suspected tumours. The clinical application of ultrasound technology is summarised well by colleagues [9], who note the ability of ultrasound being able to categorise the thickness of skin tumours to determine whether the patient may warrant non-surgical therapies, such as brachytherapy. There is also the scope for it to allow for the streamlining of pre-surgical procedures, such as identifying the size and site of the excision, as well as the type of closure. We would stress that rather than being used as a sole diagnostic tool, ultrasound should be used alongside clinical examination and histopathological findings to guide the clinician to a definitive diagnosis and management plan (Table 1). 
Table 1 Summary of the appearance of skin tumours

\begin{tabular}{lll}
\hline & Echoicity & Defining features \\
\hline Basal cell carcinoma & Hypoechoic & $\begin{array}{l}\text { Oval-shaped } \\
\text { Well-defined }\end{array}$ \\
& & $\begin{array}{l}\text { Hyperechoic spots } \\
\text { So hyperechoic spots }\end{array}$ \\
Melanoma & Hypoechoic & $\begin{array}{l}\text { Fusiform-shaped } \\
\text { Increased vascularity }\end{array}$ \\
\hline
\end{tabular}

\section{Keratinocyte skin cancers}

Basal cell carcinomas (BCC) are hypoechoic structures, oval-shaped and are well-defined [5]. Artefacts may also appear as "angles at the bottom", where dilated vessels and giant cells become inflamed, causing a hypoechoic band at the bottom; and as a "blurry tumour" due to inflamed sebaceous glands. Ultrasound in superficial and nodular BCCs strongly correlates with histopathological measurements post-excision, as it accurately predicted which tumours had subclinical extension beyond a $4 \mathrm{~mm}$ margin (48 out of 50 cases) [10].

Squamous cell carcinomas (SCC) may present as welldefined hypoechoic lesions without the hyperechoic spots seen in BCCs [5].

Broadly in BCC and SCC, ultrasound enhances precision in Mohs surgery when assessing subclinical extension in larger tumours $\left(>1.74 \mathrm{~cm}^{2}\right)$ but is not as sensitive in small tumours in detecting smaller areas of extension. Limitations arise when there can be overestimation of tumour size due to inflammatory infiltrates creating hypoechoic extensions. It may also be difficult to view hyperkeratotic SCC lesions on high-frequency ultrasound and a frequency of $13-15 \mathrm{MHz}$ may be required to visualise reticular dermal extensions in BCCs [10].

Colleagues [11] also aimed to investigate how ultrasound could determine tumour margins and whether it would be successful in being able to determine the length and width of lesions in Mohs surgery. Ultrasound images were taken on the BCC and SCC tumours in longitudinal and transverse planes before excision and between Mohs stages. In the paired $t$ tests, there were no significant differences between clinical and ultrasound widths $(t=-1.324, p=0.20)$ or lengths $(t=-1.093, p=0.29)$. For comparison of clinical evaluation and ultrasound in terms of tumour type, there was no significant difference between the widths and lengths of $\operatorname{BCC}(t=-1.307, p=0.23 ; t=-1.389, p=0.20)$ and SCC $(t=-0.342, p=0.73 ; t=0.427, p=0.68)$.

\section{Melanoma}

Melanomas are well-defined as fusiform hypoechoic lesions and have pronounced vascularity locally indicating malignant potential, but they cannot be distinguished clinically from benign melanocytic tumours. High-frequency ultrasound can discriminate melanomas less than or more than $1 \mathrm{~mm}$ in depth, which is a crucial factor when considering sentinel lymph node biopsy [5]. It can also detect in-transit ( $\geq 2 \mathrm{~cm}$ from the primary tumour), satellite ( $<2 \mathrm{~cm}$ from the primary tumour) and nodal metastases [12]. Ultrasound correlates strongly with histological thickness when assessing melanoma thickness [3]. High-frequency ultrasound $(20 \mathrm{MHz})$ correlates with larger melanomas $(>0.76 \mathrm{~cm})$ in preoperative staging, but not as accurate in thinner melanomas. If a higher frequency of $75 \mathrm{MHz}$ is used, it correlates strongly with lesions that have an average Breslow thickness of $0.4 \mathrm{~mm}$ [10].

A Cochrane review [13] aimed to determine the diagnostic accuracy of ultrasound in skin cancer. In detecting melanoma, sensitivities for qualitative characteristics (hyperechoic, homogenous, and well-defined) were at least $83 \%$ (95\% CI 75-90\%). There was $100 \%$ sensitivity (95\% CI 80-100\%) for quantitative assessments However, the authors noted that studies were poorly reported and not all the studies were designed to be diagnostic test accuracy studies, which resulted in the authors having to estimate the sensitivity and specificity in half of the studies using image descriptions. The authors were also concerned that up to $22 \%$ of lesions, including melanomas, were excluded in studies using qualitative interpretation of ultrasound as they could not be visualised.

A Cochrane review [14] investigated the accuracy of ultrasound in detecting nodal metastases in cutaneous invasive melanoma prior to sentinel lymph node biopsy (SLNB). The summary sensitivity for ultrasound to detect regional modal metastases before SLNB was 35.4\% (95\% CI 17.0-59.4\%) and specificity was $93.9 \%$ (95\% CI $86.1-97.5 \%)$. When ultrasound was combined with fineneedle aspiration cytology (FNAC), it yielded a summary sensitivity of $18.0 \%$ (95\% CI 3.58-56.5\%) and a specificity of $99.8 \%$ (95\% CI $99.1-99.9 \%$ ). For PET-CT prior to SLNB, the sensitivity was $10.2 \%$ (95\% CI 4.31-22.3\%) and the specificity was $96.5 \%$ (95\% CI 87.1-99.1\%). The authors hypothetically deduced that if there were 237 individuals with nodal metastases out of 1000 melanoma patients eligible for SLNB, ultrasound with FNAC could identify 43 patients with metastases and avoid SLNBs. There were limited data for the accuracy of primary and re-staging of melanoma.

The results do not completely support the use of ultrasound in detecting nodal metastases. There is relatively less sensitivity when ultrasound is combined with FNAC than ultrasound alone. Both have greater sensitivity than PET-CT. The authors noted that the assessing the thickness of thin tumours could be overestimated in $15 \%$ and underestimated in thick tumours in $7 \%$. 


\section{Vascular lesions}

Whilst there is a range of evidence supporting a variety of dermatological lesions, the evidence of diagnostic ultrasound in vascular lesions is relatively scarce.

Bandera et al. [15] noted that, ultrasound can identify infantile haemangioma $(\mathrm{IH})$, congenital haemangioma $(\mathrm{CH})$, and Kaposiform hemangioendothelioma, which are haemangioma variants. The latter appears as a poorly defined, large tumour which indicates heterogeneity in echogenicity, and it is a moderately to highly vascularised lesion. Like IH, there may also be calcifications.

The ultrasound appearance of IH varies on the phase of haemangioma in terms of vascularity and echogenicity. During a proliferative phase, an IH appears as a hypoechoic and hyperechoic lesion, whereas in the involutive phase, echogenicity is increased with a decrease in size and reduction in blood vessel density and size. Anechoic lacunar lesions within the lesion are correlated to sinuous blood vessels.

$\mathrm{CH}$ also appears to present similarly to $\mathrm{IH}$ as welldefined, highly vascularised tumours. $\mathrm{CH}$ can be further classified according to their clinical evolution. Rapidly involuting congenital haemangiomas (RICH) present as uniformly hypoechoic structures, whereas non-involuting congenital haemangiomas (NICH) and partially involuting congenital haemangioma (PICH) are hyperechoic tumours; their blood vessels are tubular hypoechoic structures. In $\mathrm{CH}$, particularly in RICH, calcifications may be present. Like $\mathrm{IH}$, there are also arterial and venous flows, and arteriovenous shunts present. Venous flows are more predominant in $\mathrm{NICH}$. There are also venous thrombi in $\mathrm{CH}$ that are not present in $\mathrm{IH}$.

\section{Benign lesions}

\section{Cysts}

An epidermal cyst appears [12] as a round, anechoic structure in the dermis or subcutaneous tissue, if the cyst is intact. During the inflammatory and post-rupture phases, colour Doppler ultrasound may demonstrate increased blood flow. With ultrasound, the authors acknowledge the evidence that it significantly improves the sensitivity and specificity for epidermal cyst diagnosis.

Ultrasound can visualise pilonidal cysts, which are in the inter-gluteal region. On ultrasound [5], they appear as ovalshaped hypoechoic pseudocystic structures. The cysts can be found in the dermis and subcutaneous structures with hyperechoic lines that reflect the hair. Inflammation may demonstrate enhanced peripheral vascularity. The authors note the utility of ultrasound in detecting the extent of the cysts, as well as detecting axis and supporting surgical planning.

Trichilemmal cysts may also be detected, which originate from the outer sheath of hair follicles. They are commonly found in middle-aged women and on the scalp. Clinical presentation may be a lump with associated focal alopecia. Ultrasound may demonstrate a well-defined, anechoic, or hypoechoic structure in the dermis and hypodermis; the cyst be single or multiple in nature. Unlike epidermal cysts, they do not demonstrate a punctum, but may show a target-like structure with a hyperechoic centre containing hair tracts or calcium deposits and a hypoechoic rim. [5]

\section{Pilomatrixoma}

The versatility of ultrasound in detecting benign tumours is demonstrated in pilomatrixomas [12]. The tumours have irregular contours and may demonstrate red to purple discolouration. Ultrasound will show a target lesion that has a hypoechoic rim and a hyperechoic centre. Hyperechoic spots, associated with calcification, may be seen in the centre of the lesion in most cases, and low-velocity arterial and venous vessels are seen with colour Doppler ultrasound. With a potential misdiagnosis rate of up to 56\% [5], the versatility and non-invasive nature of ultrasound is underlined as a potential medium to prevent further complications from pilomatrixomas.

\section{Dermatofibroma}

On ultrasound [5], dermatofibromas appear as oval-shaped heterogenous or hypoechoic structures. Occasionally, they may appear as both in the dermis, and less frequently in the upper hypodermis. Colour Doppler ultrasound may reveal a variable degree of vascularity from hypo-vascular to prominent blood flow.

The accuracy of ultrasound in detecting localised lesions of the skin was investigated [16]. It was determined to be a useful tool in the diagnosis of skin lesions. The referring diagnosis was correct in $73 \%$ of lesions, and the addition of ultrasound enhanced the correctness to $97 \%(p<0.001)$. The overall sensitivity stood at $99 \%$ (95\% CI 98.9-99.5\%), with a $100 \%$ specificity (95\% CI 96.4-99.9\%) and a 99\% diagnostic certainty (95\% CI 99.0-99.5\%). Ultrasound yielded higher correct diagnoses in every diagnostic category compared to the diagnosis at referral and was particularly significant for yielding a correct diagnosis for benign nonvascular lesions (96\% and 80\%, respectively) in 1998 lesions. 


\section{Inflammatory dermatoses}

A systematic review [17] investigated the role of ultrasound in nail disease in patients with psoriasis and psoriatic arthritis. When compared to healthy controls, there were more nail lesions in patients with psoriasis and psoriatic arthritis (10\%; 48.8-77.8\%; 46-54\%, respectively). A similar pattern emerged for patients with clinical nail disease: there were more lesions detected with ultrasound $(57 / 101 ; 56.4 \%)$ compared to clinical assessment $(6 / 68 ; 8.8 \%) ; p<0.0001$. Statistical analysis yielded favourable similarities between ultrasound and clinical assessment for nail disease (kappa value $=0.79$ for psoriatic arthritis patients and controls; $p<0.001)$. The authors also reported that patients with psoriasis and/or psoriatic arthritis who had an ultrasound assessment had an increased thickness of the nail plate, bed and matrix compared to controls. For resistive index (RI) measurements, psoriasis patients presented with statistically higher nail-fold vessel values compared to healthy controls.

The authors of a review [18] found trials focusing on monitoring lesional structures, blood flow and dermal thickness in localised scleroderma. The results highlighted decreased dermal thickness and hyperaemia, and after treatment, the increased echogenicity of active lesions disappeared. The authors noted a study which demonstrated the potential for ultrasound to differentiate the stages of localised scleroderma: moderate or severe sclerosis of localised scleroderma as well as active lesions would demonstrate hyperechogenicity, correlating with histology; and atrophic lesions would demonstrate hypoechogenicity.

Skin involvement in systemic scleroderma has also been reviewed [19]. The authors explored a range of ultrasound measurements vascularity against the global and site-specific modified Rodnan thickness score (MRSS). For correlation between ultrasound skin thickness and the MRSS in 5 skin sites, there was a mild to moderate positive correlation $(r=0.37[P<0.001]$ to $0.72[P<0.001])$ in 4 studies. In another study, there was a significant positive correlation in the fingers $(r=0.698[P<0.001]$ and $\mathrm{rs}=0.645[P<0.001])$ for the right and left sides, respectively, but was not reproduced in 2 other studies. It is important to note the inconsistency and variation in the skin sites across the studies. Whilst there was excellent inter-rater and intra-rater reproducibility, this was only assessed by a small number of studies. The authors also note that there were no evaluations of criterion validity of ultrasound assessment of the skin. Ultimately, it was concluded, it was difficult to lend support to the use of ultrasound in skin assessment in systemic scleroderma due to data heterogeneity.

\section{Conclusion}

Advancements in ultrasound technology have paved the way for this modality to have an increasing presence in diagnostic dermatology and to provide the dermatologist with a useful adjunct alongside their own clinical assessment. The purview of ultrasound in practice is broad, with a particular emphasis on skin cancers. Ultrasound does fall short in demonstrating efficacy for vascular lesions. Therefore, it should be used as an additional tool to help with the diagnosis of skin lesions, but challenges remain in terms of operator competency and inter-operator discrepancies.

\section{References}

1. Hofer M (1999) Ultrasound teaching manual: the basics of performing and interpreting ultrasound scans. George Thieme, Leipzig

2. de Barcaui EO, Carvalho ACP, Lopes FPPL, Piñeiro-Maceira J, Barcaui CB (2016) High frequency ultrasound with color Doppler in dermatology. An Bras Dermatol 91:262-273. https://doi.org/ 10.1590/abd1806-4841.20164446

3. Ulrich J, Voit MS, Jenderka K-V, Voit C (2014) Sonographic diagnostics in dermatology. JDDG J Dtsch Dermatol Ges 12:10831099. https://doi.org/10.1111/ddg. 12525

4. Mlosek RK, Malinowska S (2013) Ultrasound image of the skin, apparatus and imaging basics. J Ultrason 13(53):212

5. Wortsman X (2013) Ultrasound in dermatology: why, how, and when? Semin Ultrasound CT MRI Small Parts Ultrasound 34:177-195. https://doi.org/10.1053/j.sult.2012.10.001

6. Wortsman X, Alfageme F, Roustan G, Arias-Santiago S, Martorell A, Catalano O, di Santolo MS, Zarchi K, Bouer M, Gonzalez C, Bard R, Mandava A, Gaitini D (2016) Guidelines for performing dermatologic ultrasound examinations by the DERMUS Group. J Ultrasound Med 35:577-580. https://doi.org/10.7863/ultra.15. 06046

7. Trelles MA, Martínez-Carpio PA (2014) Attenuation of acne scars using high power fractional ablative unipolar radiofrequency and ultrasound for transepidermal delivery of bioactive compounds through microchannels. Lasers Surg Med 46:152-159. https://doi. org/10.1002/lsm.22224

8. Lucas VS, Burk RS, Creehan S, Grap MJ (2014) Utility of highfrequency ultrasound: moving beyond the surface to detect changes in skin integrity. Plast Surg Nurs 34:34-38. https://doi. org/10.1097/PSN.0000000000000031

9. Catalano O, Roldán FA, Varelli C, Bard R, Corvino A, Wortsman X (2019) Skin cancer: findings and role of high-resolution ultrasound. J Ultrasound 22:423-431. https://doi.org/10.1007/ s40477-019-00379-0

10. Kleinerman R, Whang TB, Bard RL, Marmur ES (2012) Ultrasound in dermatology: principles and applications. J Am Acad Dermatol 67:478-487. https://doi.org/10.1016/j.jaad.2011.12.016

11. Marmur ES, Berkowitz EZ, Fuchs BS, Singer GK, Yoo JY (2010) Use of high-frequency, high-resolution ultrasound before mohs surgery. Dermatol Surg 36:841-847. https://doi.org/10.1111/j. 1524-4725.2010.01558.x

12. Wortsman X (2012) Common applications of dermatologic sonography. J Ultrasound Med 31:97-111. https://doi.org/10.7863/jum. 2012.31.1.97

13. Dinnes J, Bamber J, Chuchu N, Bayliss SE, Takwoingi Y, Davenport C, Godfrey K, O’Sullivan C, Matin RN, Deeks JJ, Williams 
HC, Group, CSCDTA (2018) High-frequency ultrasound for diagnosing skin cancer in adults. Cochrane Database Syst Rev. https:// doi.org/10.1002/14651858.CD013188

14. Dinnes J, di Ruffano LF, Takwoingi Y, Cheung ST, Nathan P, Matin RN, Chuchu N, Chan SA, Durack A, Bayliss SE, Gulati A, Patel L, Davenport C, Godfrey K, Subesinghe M, Traill Z, Deeks JJ, Williams HC, Group, CSCDTA (2019) Ultrasound, CT, MRI, or PET-CT for staging and re-staging of adults with cutaneous melanoma. Cochrane Database Syst Rev. https://doi.org/10.1002/ 14651858.CD012806.pub2

15. Bandera AIR, Sebaratnam DF, Rodríguez MF, de Laguna RL (2020) Cutaneous ultrasound and its utility in pediatric dermatology: part II-developmental anomalies and vascular lesions. Pediatr Dermatol 37:40-51. https://doi.org/10.1111/pde.13897

16. Wortsman X, Wortsman J (2010) Clinical usefulness of variablefrequency ultrasound in localized lesions of the skin. J Am Acad Dermatol 62:247-256. https://doi.org/10.1016/j.jaad.2009.06.016

17. Mendonça JA, Aydin SZ, D'Agostino M-A (2019) The use of ultrasonography in the diagnosis of nail disease among patients with psoriasis and psoriatic arthritis: a systematic review. Adv Rheumatol 59:41. https://doi.org/10.1186/s42358-019-0081-9

18. Lis-Święty A, Janicka I, Skrzypek-Salamon A, Brzezińska-Wcisło L (2017) A systematic review of tools for determining activity of localized scleroderma in paediatric and adult patients. J Eur Acad Dermatol Venereol 31:30-37. https://doi.org/10.1111/jdv.13790

19. Santiago T, Santiago M, Ruaro B, Salvador MJ, Cutolo M, da Silva JAP (2019) Ultrasonography for the assessment of skin in systemic sclerosis: a systematic review. Arthritis Care Res 71:563-574. https://doi.org/10.1002/acr.23597

Publisher's Note Springer Nature remains neutral with regard to jurisdictional claims in published maps and institutional affiliations. 